\section{AS IMPLICAÇÕES DO COACHING SCIENCE NOS ESPORTES DE AVENTURA: UM ESTUDO DE REVISÃO SISTEMÁTICA}

\author{
COACHING SCIENCE IMPLICATIONS IN ADVENTURE SPORTS: A \\ SYSTEMATIC REVIEW STUDY
}

\section{LAS IMPLICACIONES DEL COACHING SCIENCE EN LOS DEPORTES DE AVENTURA: UN ESTUDIO DE REVISIÓN SISTEMÁTICA}

\author{
Tayná Iha*, Vitor Ciampolini*, Vinicius Zeilmann Brasil*, \\ Michel Milistetd*
}

\begin{abstract}
Palavras chave: Treinador Esportivo. Esporte de Aventura. Revisão Sistemática.

Resumo: O objetivo deste estudo foi analisar a produção científica sobre o coaching nos esportes de aventura, de modo a identificar as características dos artigos, os direcionamentos metodológicos e as tendências temáticas. Para a obtenção dos artigos foi realizada uma revisão sistemática segundo as orientações do ENTREQ e PRISMA. Foram selecionados 24 artigos indexados nas bases de dados SciELO, SPORTDiscus, Web of Science, PubMed e PsycINFO. Os artigos incluídos foram, em sua maioria, publicados nos últimos cinco anos e originários do Reino Unido. O método qualitativo foi o mais utilizado por meio da técnica de entrevista semiestruturada. Já as tendências temáticas dividiram-se em: atuação e desenvolvimento do treinador de esportes de aventura. Conclui-se que as características do ambiente de prática (ex.: risco e instabilidade) é o que influencia diretamente a atuação e o desenvolvimento do treinador. Apesar desta área ser recente, acredita-se que ela já apresenta conhecimentos de base para estudos futuros.
\end{abstract}

Keywords: Sports Coach. Adventure Sport. Systematic Review.

Palabras clave: Entrenador Deportivo. Deporte de Aventura. Revisión Sistemática.

Abstract: This study analyzes scientific articles on coaching in adventure sports in order to identify their characteristics, methodological foci, and thematic trends. We performed a systematic review in accordance with ENTREQ and PRISMA guidelines to select 24 articles indexed on Scielo, SPORTDiscus, Web of Science, PubMed, and PsycINFO. Most of the articles selected had been published in the last five years and the authors were from the UK. Qualitative methods were used more frequently through semi-structured interviews. Thematic trends were divided into adventure sports coaches' work and development. We concluded that features of the environment of practice - e.g., risk and instability - are the main factor influencing coaches' performance and development. Despite being a recent field, the body of knowledge published supports future studies to be conducted.

Resumen: El objetivo de este estudio fue analizar la producción científica sobre el coaching en los deportes de aventura, con fin de identificar las características de los artículos, las directrices metodológicas y las tendencias temáticas. Para obtener los artículos, se realizó una revisión sistemática de acuerdo con las pautas de ENTREQ y PRISMA. Se seleccionaron 24 artículos indexados en las bases de datos Scielo, SPORTDiscus, Web of Science, PubMed y PsycINFO. La mayoría de los artículos incluidos fueron publicados en los últimos cinco años y se originaron en el Reino Unido. El método más utilizado fue el cualitativo, mediante entrevistas semiestructuradas. Las tendencias temáticas se dividieron en: actuación y desarrollo del entrenador de deportes de aventura. Se concluye que las características del entorno de práctica (riesgo e inestabilidad) es lo que influye directamente en el desempeño y desarrollo del entrenador. Aunque es un área reciente, se cree que ya presenta conocimientos de base para estudios futuros.
*Universidade Federal de Santa Catarina. Florianópolis, SC, Brasil. E-mail:

taynaiha@gmail.com; vciampolini@gmail.com; vzbrasil@hotmail.com

Recebido em: 15-08-2019 Aprovado em: 15-02-2020 Publicado em: 17-03-2020 (c) (i) (8) Licence 


\section{INTRODUÇÃO}

A complexidade inerente ao coaching no contexto esportivo é um aspecto constantemente destacado na literatura específica que busca explorar as particularidades e elementos constituintes desse processo (BOWES; JONES, 2006; CUSHION, 2007). Por definição, o processo de coaching compreende as ações do treinador na organização, treinamento e competição de atletas a partir do objetivo estabelecido a curto, médio e longo prazo (COTÉ et al., 1995; ABRAHAM; COLLINS; MARTINDALE, 2006). Neste processo, o coaching é influenciado pelas características pessoais do treinador e dos atletas, pelo desenvolvimento dos atletas e por fatores contextuais (COTÉ et al., 1995). Assim, pode-se compreender o coaching pela metáfora da orquestração (orchestration), a qual apresenta o treinador como aquele que rege de forma coordenada suas ações, lidando com as variáveis do ambiente para alcançar objetivos próprios, dos atletas e da equipe (JONES; WALLACE, 2005).

No que se refere especificamente ao coaching nos esportes de aventura (EA), a complexidade adicional às ações do treinador parece estar ligada à instabilidade do ambiente de prática, seja ele terrestre (rua e montanha) aquático (mar e lago) ou aéreo (ar) (OLIVERA-BETRÁN; OLIVERA-BETRÁN, 1995; COLLINS; COLLINS, 2015a). Além da competência técnica necessária para manusear os equipamentos normalmente utilizados, é responsabilidade do treinador de esportes de aventura (TEA) considerar a instabilidade e risco do ambiente de prática para proporcionar segurança, bem-estar e integridade física própria e do praticante (COLLINS; COLLINS, 2012; 2013; 2015a). Apesar das investigações conduzidas acerca do TEA auxiliarem na compreensão das especificidades relacionadas ao coaching nessas modalidades, o corpo de conhecimento relacionado ao treinador esportivo dos esportes tradicionais ainda parece ser substancialmente superior.

Por exemplo, quando se trata de revisões de literatura acerca do treinador esportivo, diversos estudos têm sido realizados nos últimos anos. Gilbert e Trudel (2004) conduziram a primeira revisão sobre a temática, realizando um levantamento sobre os estudos publicados entre 1970 e 2001. Mesmo que os autores tenham revisado 610 estudos, o TEA não foi mencionado e nenhuma modalidade dos EA se fez presente na lista de esportes que apareceram em pelo menos $5 \%$ dos estudos (GILBERT; TRUDEL, 2004). Com o passar dos anos as revisões têm apresentado foco cada vez mais específico na busca e análise da produção científica, incluindo a efetividade de programas de formação de treinadores (LANGAN; BLAKE; LONSDALE, 2013), as percepções de treinadores sobre as estratégias de ensino propostas em programas de formação (CIAMPOLINI et al., 2019) e a aprendizagem informal (no dia a dia) e não-formal (em cursos) de treinadores (WALKER; THOMAS; DRISKA, 2018). Embora se reconheça as relevantes contribuições dessas revisões, destaca-se que as investigações no âmbito do coaching nos EA não têm sido considerados como objetos de análise até o presente momento.

Do mesmo modo que a revisão de literatura inicial conduzida por Gilbert e Trudel (2004) contribuiu com a obtenção de um panorama da produção científica sobre o treinador esportivo, destaca-se a importância de um estudo de revisão que 
revele os direcionamentos das investigações sobre o coaching nos EA. Nos últimos anos, a popularização dos EA tem aumentado consideravelmente, repercutindo no crescente número de praticantes e treinadores (THORPE; WHEATON, 2011). Destaca-se ainda que, em 2020, além dos EA que já estavam presentes na edição dos Jogos Olímpicos de Verão de 2016 (Canoagem, Remo e Vela), outras novas modalidades serão incluídas como a Escalada, o Surfe e o Skate (MOTA; SEDA, 2016). Portanto, considerando as evidentes demandas para a prática esportiva e intervenção profissional no âmbito dos EA, assim como a necessidade de identificar as tendências nessa área, o objetivo deste estudo é realizar uma análise da produção científica sobre o coaching nos EA de modo a identificar as características dos estudos, os direcionamentos metodológicos e tendências temáticas, por meio de procedimentos de revisão sistemática.

\section{MÉTODO}

A presente pesquisa foi realizada segundo o guia de revisão sistemática específico à ciência do coaching (BENNIE et al., 2017) e as recomendações PRISMA (GALVÃO; PANSANI; HARRAD, 2015) de modo a identificar as publicações científicas acerca do TEA. A busca dos artigos foi realizada no mês de junho de 2018 e consistiu nos passos apresentados a seguir.

\subsection{ESTRATÉGIAS DE BUSCA}

A busca dos artigos se deu nas bases de dados Scielo, SPORTDiscus, Web of Science, PubMed e PsycINFO. A sintaxe utilizada para a busca foi construída com termos de dois grupos: esporte e treinador. Para combinar os grupos utilizouse o operador booleano "AND" e para buscar mais de um termo dentro do grupo utilizou-se o operador booleano "OR", além disso utilizou-se aspas para os termos longos afim de evitar que estes fossem buscados como palavras distintas. A busca nas bases de dados foi realizada com ambas as sintaxes (Quadro 1) e nenhum filtro foi adicionado a priori.

Quadro 1 - Construção da sintaxe para as buscas nas bases de dados.

\begin{tabular}{|l|l|l|}
\hline & \multicolumn{1}{|c|}{ PORTUGUÊS } & \multicolumn{1}{|c|}{ INGLÊS } \\
\hline \multirow{2}{*}{ ESPORTE } & $\begin{array}{l}\text { Esporte de aventura } \\
\text { Esporte ao ar livre } \\
\text { Esporte radical }\end{array}$ & $\begin{array}{l}\text { Adventure sport } \\
\text { Outdoor sport } \\
\text { Extreme sport }\end{array}$ \\
\hline \multirow{2}{*}{ TREINADOR } & $\begin{array}{l}\text { Treinador } \\
\text { Instrutor }\end{array}$ & $\begin{array}{l}\text { Coach } \\
\text { Instructor }\end{array}$ \\
\hline \multirow{2}{*}{ SINTAXE } & $\begin{array}{l}\text { treinador OR instrutor AND "esporte de } \\
\text { aventura" OR "esporte ao ar livre" OR } \\
\text { "esporte radical" }\end{array}$ & $\begin{array}{l}\text { coach OR instructor AND "adventure } \\
\text { sport" OR "outdoor sport" OR "extreme } \\
\text { sport" }\end{array}$ \\
\hline
\end{tabular}

Fonte: Próprio autor

Ao compreender que artigos envolvendo o tema poderiam tratar de uma única modalidade de aventura e não usar o termo "esportes de aventura", fez-se uma busca adicional para minimizar a perda destes. Para esta busca, selecionou-se todos os EA 
previstos para os Jogos Olímpicos de Verão de 2020 (canoa, caiaque, remo, vela, windsurfe, escalada e surfe) e formou-se sintaxes com os termos referentes aos treinadores. Para cada esporte fez-se uma sintaxe e as buscas foram individuais, as quais foram realizadas nas mesmas bases da busca principal.

\subsection{SELEÇÃO DOS ARTIGOS}

Ao todo, foram encontrados 298 artigos na busca principal, os quais foram incluídos no software EndNote versão X7. Os artigos duplicados foram excluídos e, em seguida, fez-se a conferência da lista para exclusões manuais quando necessário. O processo de seleção dos artigos (Figura 1) foi realizado por dois revisores independentes e, quando houve divergência das informações, a decisão a ser tomada se deu por meio de reunião de consenso. Quando essa divergência permaneceu, houve a intervenção de um terceiro autor para a decisão final. A primeira etapa consistiu na leitura dos títulos, seguida pela leitura dos resumos e, por último, a leitura dos artigos na íntegra.

Figura 1 - Fluxograma da seleção dos artigos para a revisão sistemática.

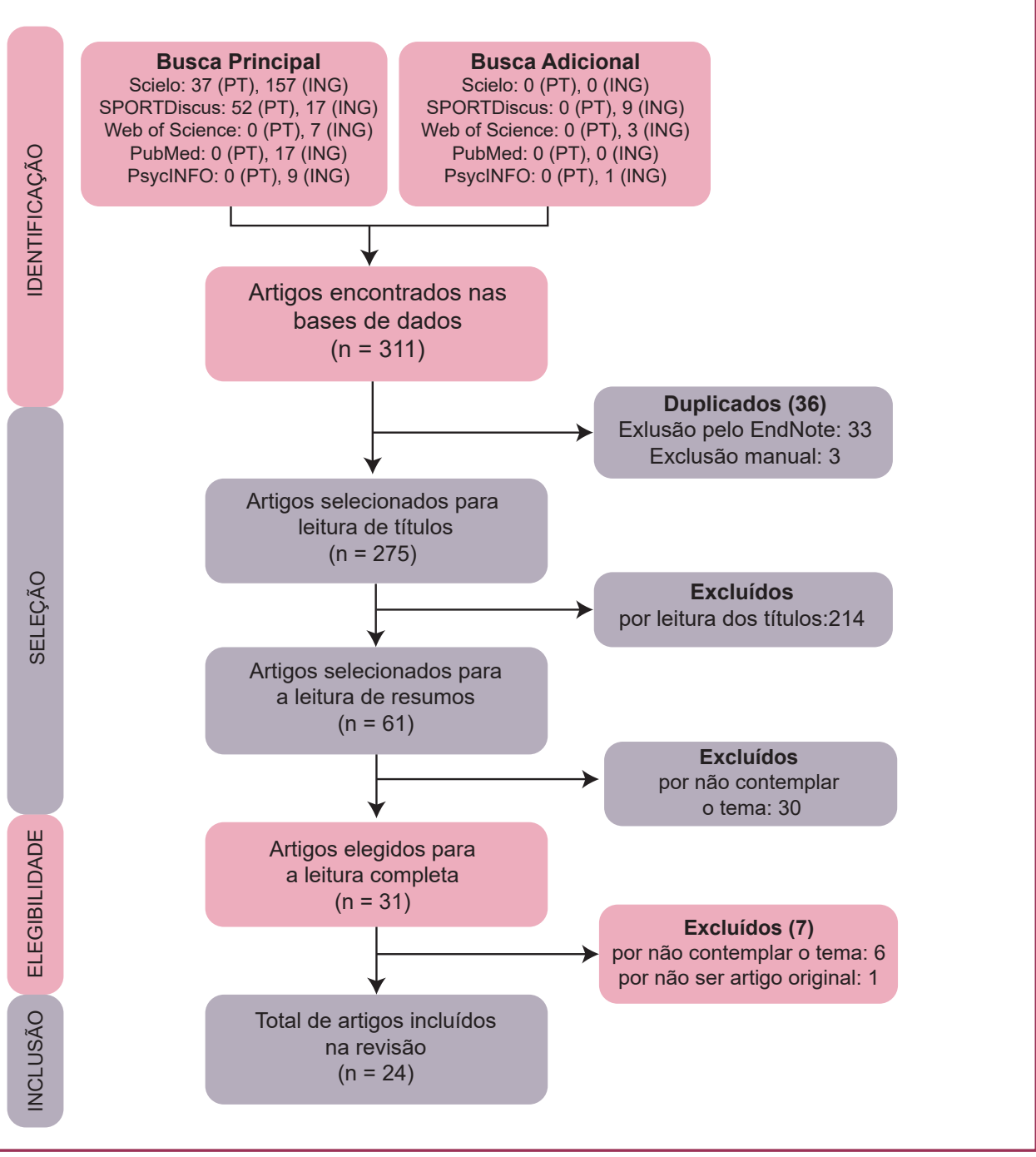

Fonte: Próprio autor.

Legenda: $\mathrm{PT}$ = português; ING = inglês. 
Para a seleção foram adotados três critérios de inclusão: contemplar o TEA como tema central, estar publicado na língua portuguesa ou inglesa e ser artigo original. Foram adotados como critérios de exclusão: contemplar instrutores como tema central, artigos de revisão ou validação de instrumento e não ter acesso ao artigo na íntegra. Os artigos que investigaram instrutores não foram incluídos na revisão, uma vez que a sua atuação não se configura como um TEA. Porém, a inclusão do termo "instrutor" na sintaxe se deu com o intuito de evitar a perda de artigos que poderiam ter usado esta terminologia para se referir aos TEA, de modo que a leitura na íntegra realizada pelos autores identificaria essa questão. A partir da busca nas bases de dados e da aplicação dos critérios supracitados foram selecionados 24 artigos para esta revisão.

\subsection{EXTRAÇÃO E ANÁLISE DOS DADOS}

Esta etapa foi realizada por dois pesquisadores de forma independente e incluiu reuniões de consenso quando necessário. Os dados extraídos do corpo dos artigos foram: autores, ano de publicação, país em que ocorreu a investigação, modalidades esportivas investigadas, método utilizado, tema central e contexto de formação do treinador estudado.

\subsection{AVALIAÇÃO DA QUALIDADE DOS ARTIGOS}

Para a avaliação da qualidade dos artigos incluídos na revisão, utilizou-se o checklist COREQ (TONG; SAINSBURY; CRAIG, 2007) que contempla 32 itens distribuídos em três categorias de avaliação: equipe de pesquisa e reflexividade; desenho do estudo e; análise e resultados. Este procedimento foi adotado para permitir aos leitores a avaliação da fidedignidade e confiabilidade das informações apresentadas. Toda a avaliação foi conduzida por dois autores de maneira conjunta.

Devido ao instrumento se propor a avaliar somente artigos que utilizam entrevistas e grupo-focal como instrumento, três dos 24 artigos inclusos não participaram desta avaliação (MILLAR; OLDHAM; DONOVAN, 2011; KIOSOGLOUS; VIDIC, 2017; MILLAR et al., 2017). Dos 21 artigos avaliados, quase todos compreenderam mais do que $50 \%$ dos itens, um ficou abaixo dessa marca e 10 compreenderam mais do que $65 \%$. Para compreender a avaliação individual dos artigos a partir das três dimensões do checklist, consultar a tabela 1.

Das três dimensões avaliadas, a "análise e resultados" $(80,42 \%)$ foi a mais contemplada pelos artigos, seguida pelo "desenho do estudo" (62,50\%) e "equipe de pesquisa e reflexividade" (39,52\%). Acredita-se que a "análise e resultados" foi a mais contemplada devido à cultura acadêmica estabelecida sobre a importância da apresentação das informações relacionadas aos dados nas pesquisas qualitativas. Por outro lado, a "equipe de pesquisa e reflexividade" (ex.: qual é a ocupação do investigador no período do estudo? Quais são as experiências e treinamentos do pesquisador?) parece ser pouco explorada nos estudos, sendo a única dimensão a receber o score zero. Sugere-se que estudos futuros detalhem mais esta dimensão para dar suporte à fidedignidade da pesquisa qualitativa. 
Tabela 1 - Avaliação dos artigos segmentada pelas dimensões do checklist COREQ (TONG; SAINSBURY; CRAIG, 2007).

\begin{tabular}{|c|c|c|c|c|}
\hline \multirow[b]{2}{*}{ ARTIGOS } & \multicolumn{3}{|c|}{ DIMENSÕES (\%) } & \multirow{2}{*}{$\begin{array}{c}\text { TOTAL } \\
(\%)\end{array}$} \\
\hline & $\begin{array}{c}\text { Equipe de pesquisa } \\
\text { e reflexividade }\end{array}$ & $\begin{array}{l}\text { Desenho do } \\
\text { estudo }\end{array}$ & $\begin{array}{l}\text { Análise e } \\
\text { resultados }\end{array}$ & \\
\hline Brasil et al. (2016) & 25 & 60 & 77,8 & 56,3 \\
\hline Brasil et al. (2017) & 12,5 & 80 & 100 & 68,8 \\
\hline Christian, Berry e Kearney (2017) & 25 & 53,3 & 66,7 & 50 \\
\hline Collins, Collins e Carson (2016) & 75 & 46,7 & 88,9 & 65,6 \\
\hline Collins, Collins e Grecic (2015) & 75 & 66,7 & 66,7 & 68,8 \\
\hline Collins e Collins (2015a) & 87,5 & 60 & 88,9 & 75 \\
\hline Collins e Collins (2015b) & 62,5 & 60 & 88,9 & 68,8 \\
\hline Collins e Collins (2016b) & 75 & 53,3 & 88,9 & 68,8 \\
\hline Collins e Collins (2016a) & 62,5 & 53,3 & 88,9 & 65,6 \\
\hline Duarte e Culver (2014) & 25 & 78,57 & 77,8 & 64,5 \\
\hline Gray e Collins (2016) & 37,5 & 73,3 & 66,7 & 62,5 \\
\hline Lorimer e Holland-Smith (2012) & 12,5 & 57,1 & 66,7 & 48,4 \\
\hline Ramos, Brasil e Goda (2012) & 0 & 46,7 & 100 & 50 \\
\hline Ramos, Brasil e Goda (2013) & 25 & 73,3 & 88,9 & 65,6 \\
\hline Ramos et al. (2014) & 25 & 66,7 & 77,8 & 59,4 \\
\hline Saury e Durand (1998) & 25 & 60 & 77,8 & 56,3 \\
\hline Simon, Collins e Collins (2017) & 37,5 & 53,3 & 66,7 & 53,1 \\
\hline Correia e Bertram (2018) & 75 & 73,3 & 88,9 & 78,1 \\
\hline Mahoney et al. (2016) & 0 & 60 & 88,9 & 53,1 \\
\hline Maclellan, Callary e Young (2018) & 12,5 & 66,7 & 77,8 & 56,3 \\
\hline Pope, Penney e Smith (2018) & 50 & 71,4 & 55,5 & 61,3 \\
\hline TOTAL (\%) & 39,52 & 62,5 & 80,42 & - \\
\hline
\end{tabular}

\section{RESULTADOS}

\subsection{CARACTERÍSTICAS DAS PESQUISAS SOBRE COACHING NOS EA}

A partir da análise dos artigos selecionados (24) verificou-se que esses estudos se originaram, predominantemente, do Reino Unido (11) e do Brasil (5) (Tabela 2). Relativo ao ano de publicação dos artigos, destaca-se que o primeiro manuscrito publicado, de autoria de Saury e Durand (1998), analisou o conhecimento de treinadores experts de vela olímpica. Após um intervalo de 13 anos sem publicações na área, constatou-se um número reduzido de artigos publicados de maneira dispersa entre 2011 e 2014. Se, por um lado, de 1998 a 2014 foram publicados seis artigos envolvendo o coaching nos EA, a partir de 2015 houve um aumento exponencial de publicações sobre este tema, sendo publicados 18 artigos até o ano de 2019. Referente às modalidades esportivas contempladas nos artigos, verificou-se o predomínio de EA praticados em ambiente aquático, destacadamente: surf, caiaque, canoagem, remo e vela. 
Tabela 2 - Dados gerais dos artigos incluídos na revisão.

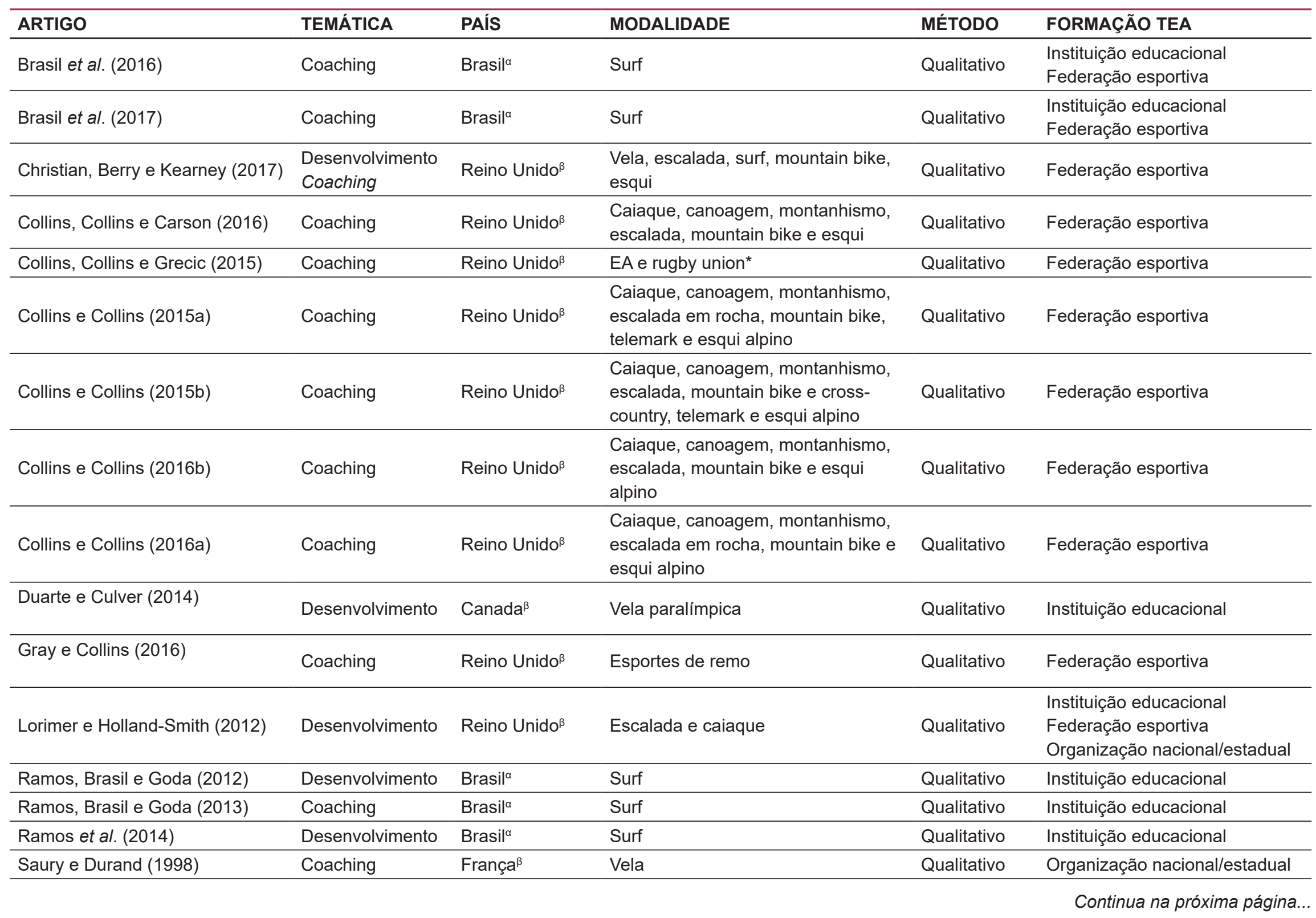

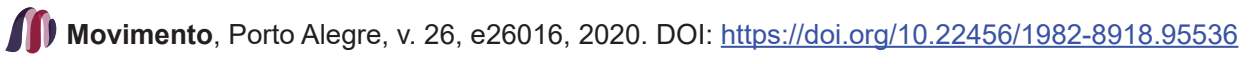


Tayná Iha, Vitor Ciampolini, Vinicius Zeilmann Brasil, Michel Milistetd

Continuação da tabela 2

\begin{tabular}{|c|c|c|c|c|c|}
\hline Simon, Collins e Collins (2017) & Coaching & Reino Unido ${ }^{\beta}$ & Esportes de remo & Qualitativo & Federação esportiva \\
\hline Correia e Bertram (2018) & Coaching & Portugal $^{\beta}$ & Surf & Qualitativo & Federação esportiva \\
\hline Mahoney et al. (2016) & Coaching & Reino Unido ${ }^{\beta}$ & Remo & Qualitativo & Federação esportiva \\
\hline Maclellan, Callary e Young (2018) & Coaching & Canada ${ }^{\beta}$ & Canoa e caiaque & Qualitativo & $\begin{array}{l}\text { Instituição educacional } \\
\text { Organização nacional/estadual }\end{array}$ \\
\hline Millar, Oldham e Donovan (2011) & Coaching & Inglaterra $^{\beta}$ & Remo & Quantitativo & $\mathrm{NI}$ \\
\hline Millar et al. (2017) & Coaching & Nova Zelândia ${ }^{\beta}$ & Remo & Quali/Quanti & $\mathrm{NI}$ \\
\hline Pope, Penney e Smith (2018) & Coaching & Nova Zelândia ${ }^{\beta}$ & Remo & Qualitativo & Instituição educacional \\
\hline Kiosoglous e Vidic (2017) & Desenvolvimento & Estados Unidos ${ }^{\beta}$ & Remo & Quantitativo & $\mathrm{NI}$ \\
\hline
\end{tabular}

\section{Fonte: Próprio autor.}

*não é um EA, mas foi utilizado a fim de comparação no artigo

Legenda: $\alpha$ (português); $\beta$ (inglês); Instituição educacional (cursos de educação superior); Federação esportiva (cursos de certificação específicos do esporte); Organização nacional/ estadual (cursos de certificação esportiva geral); $\mathrm{NI}$ (não informado). 
3.2 ABORDAGENS E PROCEDIMENTOS METODOLÓGICOS DAS PESQUISAS SOBRE COACHING NOS EA

A respeito da abordagem dos artigos analisados, a maioria $(87,5 \%)$ foi por meio de procedimentos de pesquisa qualitativa, com predomínio da utilização de técnicas de coleta de dados como a entrevista semiestruturada $(80,9 \%)$. Já os artigos orientados a partir de procedimentos quantitativos $(8,3 \%)$ priorizaram a utilização das técnicas de análise de regressão múltipla (100\%). Somente um artigo $(4,2 \%)$ utilizou a abordagem mista.

No que se refere aos treinadores pesquisados nos estudos, o contexto de atuação foi predominantemente de rendimento $(71,4 \%)$, sendo poucos envolvidos no contexto de desenvolvimento $(38,1 \%)$ e participação $(4,8 \%)$. Quanto à formação destes treinadores (Tabela 2), grande parte apresentou certificação no contexto federativo $(66,7 \%)$, seguido pela formação universitária $(42,8 \%)$ e de organizações nacionais $(14,3 \%)$. É importante destacar que todos os treinadores apresentaram formação universitária nos artigos desenvolvidos no Brasil. Por fim, treinadores experientes parecem ter sido o maior público investigado, visto o tempo de atuação elevado em diversos artigos selecionados para esta revisão.

\subsection{ENFOQUES TEMÁTICOS DOS ESTUDOS SOBRE COACHING NOS EA}

Os principais temas abordados nos artigos incluídos na revisão parecem ter dois direcionamentos temáticos principais: atuação do TEA e desenvolvimento do TEA. O primeiro tema abrange os conhecimentos e a intervenção; e a segunda compreende a aprendizagem e a trajetória de vida.

\subsubsection{Atuação do TEA}

\section{Conhecimentos}

De modo geral, os artigos nesta temática apontam pela necessidade do TEA dominar os conhecimentos específicos da modalidade praticada (contexto, equipamento, praticante). Assim, compreender as variáveis do ambiente natural e a interação entre elas parece refletir na escolha dos equipamentos, os quais também se diferenciam quanto às necessidades do praticante para uma modalidade específica dentro do esporte. Além disso, ter conhecimento sobre as ciências do esporte (tais como: Fisiologia, Psicologia, Pedagogia, entre outras) auxilia no processo de ensino-aprendizagem, nos quais os TEA buscam passar o conhecimento da melhor forma possível a seus aprendizes. Como uma forma de se envolver e gerar confiança, o treinador acaba por assumir o papel de amigo, ficando próximo de seu aluno, fortalecendo as relações interpessoais entre ambos e, assim, sendo capaz de compreender as motivações e interesses que permeiam o aluno. Conhecer os próprios objetivos, planejar-se e organizar as sessões de treino também parece ser importante para poder desenvolver comportamentos e valores, os quais suportarão a autonomia do aluno na prática da modalidade. 
Além disso, Saury e Durand (1998) introduzem o termo 'antecipação cognitiva baseada em planos flexíveis', o qual compreende os conhecimentos mencionados acima, também destacados por outros autores (RAMOS; BRASIL; GODA, 2013; BRASIL et al., 2017). Este termo se refere a leitura do ambiente de prática para adaptação a eventuais imprevistos, assim como a compreensão das experiências prévias e objetivos do praticante para a atividade. Desta forma, o TEA busca prever possíveis eventos, mudanças no ambiente e ações do praticante para diminuir a sua carga cognitiva durante o coaching.

\section{Intervenção}

A partir dos estudos liderados ao longo dos anos pelos autores Loel Collins, Dave Collins e seus respectivos colaboradores (ex.: COLLINS; COLLINS, 2015a; COLLINS; COLLINS; GRECIC, 2015; COLLINS; COLLINS; CARSON, 2016;), o termo 'julgamento profissional e tomada de decisão' (JPTD) é utilizado para se referir às habilidades pedagógicas do TEA para selecionar as estratégias de ensino adequadas às possibilidades do praticante. Desta forma, durante a prática de trabalho, é importante que o treinador seja capaz de controlar e gerenciar o tempo para facilitar o JPTD. Este processo é necessário para lidar com diversos elementos, tais como a gestão dos riscos, seleção do local de aula, objetivos e conteúdo da sessão.

Outros aspectos que se relacionam à intervenção do TEA é o contexto em que atua e as suas ações, assim como os tipos de feedback e ferramentas que utiliza para identificar a carga de treinamento dos praticantes. Complementarmente, os aspectos relacionados à interação treinador-atleta também são integrados, uma vez que para que ela ocorra e seja efetiva, é importante que o treinador crie um ambiente apropriado e conheça seu aluno. Desta forma, o treinador é capaz de gerar uma relação próxima e de confiança, além de proporcionar a autonomia do praticante.

\subsubsection{Desenvolvimento do TEA}

\section{Aprendizagem}

Apesar de a aprendizagem de TEA ocorrer por diversas fontes, as informais tendem a ser as mais valorizadas, tais como as experiências como praticante, as resoluções de problemas na prática, a reflexão e o acesso a materiais na internet, livros, revistas e filmes. Além disso, o aspecto social da aprendizagem por meio do compartilhamento de informações e observação dos pares, mentores e atletas também é destacado. Quanto à aprendizagem por meio da participação em cursos, os TEA também apontaram, de modo geral, a contribuição de capacitações e certificações oferecidas por entidades esportivas (federações e entidades nacionais) e não-esportivas (faculdades e entidades privadas). Assim, os TEA parecem compreender a aprendizagem como um processo contínuo e veem a importância de estarem abertos para aprender por diversas fontes. Porém, há valorização das fontes informais, uma vez que há o engajamento espontâneo para dominar temas específicos da modalidade que ensina, bem como pelas relações de socialização estabelecidas com os pares para contribuir na aprendizagem. 
Trajetórias de vida

A trajetória dos TEA parece se iniciar na infância, a partir do envolvimento em atividades no ambiente natural. Essas atividades geralmente são realizadas juntamente com amigos e familiares próximos, os quais parecem promover essa inserção. Além disso, o envolvimento costuma se dar em momentos de lazer, seja por envolvimento direto (ex.: ir para a praia ou parques com os pais) ou indireto (ex.: participar de eventos esportivos como espectador).

Posteriormente, o ingresso na carreira de treinador parece se dar de maneira "natural", a partir do acúmulo de experiências práticas na modalidade e o desejo por auxiliar outras pessoas. Já a permanência na carreira de treinador parece se dar pelo valor atribuído às experiências pessoais anteriores. Assim, as experiências positivas na infância, a paixão pelo esporte, o legado que pretende deixar, o estilo de vida que leva e a necessidade de manter-se envolvido com o EA que pratica são alguns dos fatores que os motivam a seguir na carreira.

\section{DISCUSSÃO}

Considerando os artigos selecionados e analisados no presente estudo, evidenciou-se um aumento no número de publicações a partir do ano de 2015. Apesar de este aumento ser impulsionado, em parte, pelo anúncio da inclusão do surf, do skate e da escalada nos Jogos Olímpicos de Verão de 2020 (MOTA; SEDA, 2016), a concentração de estudos, sobretudo, no Reino Unido sugere a influência de pesquisadores deste local nas publicações cientificas sobre o coaching nos EA, de fato, pelas iniciativas de Dave Collins e Loel Collins. Além disso, os artigos de autoria destes pesquisadores parecem ser produtos de projetos investigativos mais abrangentes, como no caso, por exemplo, de cinco artigos envolvendo participantes e métodos semelhantes (COLLINS; COLLINS, 2015a; 2015b; COLLINS; COLLINS; GRECIC, 2015; COLLINS; COLLINS, 2016b; 2016a).

Embora se reconheça a indefinição a respeito da denominação do profissional que intervém no âmbito dos EA, o termo TEA ou Adventure Sports Coach, proposto por Collins e Collins (2012), corresponde a uma possibilidade de delimitação conceitual dessa atividade profissional emergente no contexto esportivo atual. Em essência, o TEA é o profissional que tem o papel de desenvolver praticantes capazes de participar, de maneira autônoma, em suas próprias experiências esportivas de aventura, sejam elas voltadas ao lazer, como estilo de vida ou para o alto rendimento (COLLINS; COLLINS, 2015a). Portanto, apesar de reconhecer a diversidade de aspectos que influenciaram a ampliação da produção científica com foco no coaching nos EA, com base nos artigos incluídos na presente revisão constatou-se a relevante contribuição desses pesquisadores para o aumento das publicações nesta área nos últimos anos.

Referente às modalidades esportivas contempladas nos artigos analisados, predominaram os EA praticados em ambientes aquáticos e terrestres, os quais, em sua maioria, já integram um cenário esportivo de elevada estrutura organizacional e com participação em megaeventos esportivos. Por outro lado, a ausência de 
modalidades aéreas pode-se justificar pela ausência do treinador esportivo, onde a prática é iniciada por outros agentes (como praticantes mais experientes e instrutores) como por exemplo, o voo livre, o paraquedismo e o wingsuit, os quais têm sido denominados de lifestyle sports (WHEATON, 2004). Embora haja manifestações competitivas nos lifestyle sports, estes não visam a "esportivização" exacerbada e a profissionalização da prática (WHEATON, 2004), levando a ausência da necessidade do coaching para a busca de níveis mais elevados de desempenho esportivo.

Acredita-se que a predominância de estudos qualitativos para a investigação do TEA se deu pelas características deste método. Particularmente, a condução de entrevistas nos estudos sobre o coaching e sobre o TEA revelou a ênfase na análise das opiniões, sentimentos, emoções, experiências e seus significados, bem como buscou-se compreender as perspectivas do treinador sobre o "por quê", "o quê" e "como" trabalha e exerce suas funções (NELSON; GROOM; POTRAC, 2014). No caso dos estudos analisados, acredita-se que a adoção de procedimentos qualitativos revela a tendência à busca pela compreensão aprofundada do fenômeno em torno do TEA em detrimento à generalização do conhecimento produzido por meio de amostras representativas, normalmente almejado por métodos quantitativos (GÜNTHER, 2006).

Embora haja semelhanças entre os treinadores de esportes tradicionais e os TEA quanto aos conhecimentos necessários para atuação, os TEA parecem apresentar particularidades quanto aos conhecimentos profissionais relacionados ao ambiente de prática. Se por um lado nos esportes tradicionais o ambiente é estável (como, por exemplo, o ginásio para os esportes coletivos), nos EA ele é instável e imprevisível (como, por exemplo, o mar), uma vez que o espaço de prática é o meio natural (COLLINS; COLLINS, 2012). As evidências destes estudos indicam que o TEA deve dominar os conhecimentos acerca do ambiente, seja na disposição do espaço, na leitura das suas modificações e, a partir das informações interpretadas, ser capaz de se adaptar às mudanças e diminuir os riscos. Este processo foi denominado como um tipo de "antecipação cognitiva em planos flexíveis" apresentado por Saury e Durand (1998). Portanto, reforça-se a importância da experiência prévia de TEA na prática da modalidade para a compreensão do ambiente, bem como dos elementos essenciais nos EA (relação entre praticante-equipamento-ambiente) (FUNOLLET, 1995), o que se acredita facilitar o processo de ensino-aprendizagem.

Outro aspecto evidenciado nos artigos incluídos quanto à atuação do TEA, é a valorização da relação treinador-aluno em diversos EA. A qualidade dessa relação é o que define a essência do coaching, bem como os papéis que são assumidos pelo treinador (amigo, companheiro e alguém próximo do aluno) (BRASIL et al., 2017). Assumir tais papéis contribui diretamente na confiança e comprometimento estabelecido entre ambos, bem como na qualidade da atuação do treinador (JOWETT, 2017). Nos EA, a confiança recebe conotação adicional, pois o risco inerente à prática pode ser percebido de forma diferente pelo treinador e pelo próprio aluno (PAIXÃO et al., 2011; MOURA; FERREIRA; SOARES, 2016). Assim, a relação treinador-aluno parece ser fundamental para favorecer a confiança bidirecional, ou seja, para que o aluno confie nas atividades propostas e situações desafiadoras criadas pelo treinador, 
bem como para que o treinador confie nas habilidades do aluno e que este atenderá às instruções fornecidas mesmo em situações adversas.

Quanto aos estudos com foco na aprendizagem dos treinadores, ficou evidenciado a relevância da trajetória de prática esportiva em EA desses indivíduos iniciada na infância. O contato com uma determinada cultura esportiva, desde as idades iniciais, por meio do engajamento na prática, em situações significativas vinculadas à grupos sociais com interesses em comum (família, amigos, atletas, etc.) parece promover uma aprendizagem efetiva (WENGER, 2009). De fato, a valorização das experiências em contextos informais é percebida tanto por treinadores de esportes tradicionais quanto por TEA, devido ao seu caráter contextualizado e significativo (WENGER, 2009; RAMOS et al., 2011; VIRGÍLIO et al., 2017). A negociação dos significados atribuídos à estas experiências, por meio das interações sociais, ao longo da trajetória dos TEA direciona a priorização de tais contextos de aprendizagem (WENGER, 2009).

Quanto ao contexto de formação dos treinadores investigados nos artigos selecionados, destaca-se também a valorização de federações esportivas e instituições educacionais (universidades) como vias de qualificação e certificação. Segundo o International Council for Coaching Excellence, a formação de treinadores é uma responsabilidade das federações esportivas, sejam elas nacionais ou internacionais (ICCE, 2013). Assim, são elas que devem determinar o processo de capacitação e certificação para a atuação dos treinadores (ICCE, 2013). Contudo, devido à profissionalização da intervenção do treinador esportivo no Brasil e a exigência pela formação universitária (MILISTETD et al., 2014), os TEA investigados nos estudos conduzidos no Brasil apresentaram formação neste contexto. Considerando que a profissionalização do treinador não é realidade nos países dos demais artigos incluídos nesta revisão (tais como aqueles desenvolvidos no Reino Unido, Canadá, Inglaterra, Portugal e Nova Zelândia), nem todos os treinadores investigados apresentavam formação em instituições educacionais.

\section{CONSIDERAÇÕES FINAIS}

O objetivo do presente estudo foi analisar as implicações do coaching science nas publicações científicas com foco no coaching nos EA. Ao analisar artigos empíricos selecionados no presente estudo (24), percebeu-se que esta é uma área de estudo recente, iniciada de forma pouco expressiva por volta dos anos 2000 e mais difundida a partir de 2015. Esta marca se deu, sobretudo, por um grupo de pesquisadores que conduziram grandes projetos que originaram importante quantidade de artigos, contribuindo no montante de publicações na área. Embora a ciência do TEA ainda esteja em desenvolvimento, as publicações existentes já elucidam o campo, bem como trazem conhecimentos de base para o desenvolvimento de estudos futuros.

Quanto aos enfoques temáticos dos artigos analisados, constatou-se o predomínio das subáreas de atuação e desenvolvimento do treinador. A partir da apreciação destas temáticas, foi possível identificar semelhanças e peculiaridades dos TEA com treinadores de esportes tradicionais. De modo geral, o que distingue os 
dois contextos é o local de prática da modalidade e suas características. O ambiente natural, o risco, a instabilidade e as incertezas inerentes aos EA é o que influencia diretamente a atuação e o desenvolvimento do treinador. Destaca-se a importância do TEA em dominar os conhecimentos para a leitura do ambiente para que, durante a intervenção, faça constantes julgamentos e tomadas de decisão para garantir a segurança do aluno. Por fim, as experiências em ambientes naturais desde a infância parecem influenciar na valorização das experiências práticas pessoais e com os pares para a aprendizagem dos TEA.

\section{REFERÊNCIAS}

ABRAHAM, Andy; COLLINS, Dave; MARTINDALE, Russell. The coaching schematic: Validation through expert coach consensus. Journal of Sports Sciences, v. 24, n. 6, p. 549564, 2006.

BENNIE, Andrew, et al. A guide to conducting systematic reviews of coaching science research. International Sport Coaching Journal, v. 4, n. 2, p. 191-205, 2017.

BOWES, Imornefe; JONES, Robyn L. Working at the edge of chaos: Understanding coaching as a complex, interpersonal system. The Sport Psychologist, v. 20, n. 2, p. 235245, 2006.

BRASIL, Vinicius Zeilmann, et al. As ações pedagógicas para a intervenção do treinador de surf. Movimento, v. 22, n. 2, p. 403-416, 2016.

BRASIL, Vinicius Zeilmann, et al. Os conhecimentos de base para intervenção pedagógica do treinador de surf. Revista Brasileira de Educação Física e Esporte, v. 31, n. 4, p. 807817, 2017.

CHRISTIAN, Ed; BERRY, Matt; KEARNEY, Phil. The identity, epistemology and developmental experiences of high-level adventure sports coaches. Journal of Adventure Education and Outdoor Learning, v. 17, n. 4, p. 353-366, 2017.

CIAMPOLINI, Vitor, et al. Research review on coaches' perceptions regarding the teaching strategies experienced in coach education programs. International Journal of Sports Science and Coaching, v. 14, n. 2, p. 216-228, 2019.

COLLINS, Loel; COLLINS, Dave. Conceptualizing the adventure-sports coach. Journal of Adventure Education and Outdoor Learning, v. 12, n. 1, p. 81-93, 2012.

COLLINS, Loel; COLLINS, Dave. Decision making and risk management in adventure sports coaching. Quest, v. 65, n. 1, p. 72-82, 2013.

COLLINS, Loel; COLLINS, Dave. Integration of professional judgement and decision-making in high-level adventure sports coaching practice. Journal of Sports Sciences, v. 33, n. 6, p. 622-633, 2015a.

COLLINS, Loel; COLLINS, Dave. Professional judgement and decision-making in adventure sports coaching: The role of interaction. Journal of Sports Sciences, v. 34, n. 13, p. 12311239, 2015b. 
COLLINS, Loel; COLLINS, Dave. Professional judgement and decision-making in the planning process of high-level adventure sports coaching practice. Journal of Adventure Education and Outdoor Learning, v. 16, n. 3, p. 256-268, 2016b.

COLLINS, Loel; COLLINS, Dave. The foci of in-action professional judgement and decisionmaking in high-level adventure sports coaching practice. Journal of Adventure Education and Outdoor Learning, v. 17, n. 2, p. 122-132, $2016 a$.

COLLINS, Dave; COLLINS, Loel; CARSON, Howie J. "If it feels right, do it": Intuitive decision making in a sample of high-level sport coaches. Frontiers in Psychology, v. 7, p. 504, 2016.

COLLINS, Loel; COLLINS, Dave; GRECIC, David. The epistemological chain in high-level adventure sports coaches. Journal of Adventure Education and Outdoor Learning, v. 15, n. 3, p. 224-238, 2015.

CORREIA, Marco Catarino Espada Estêvão; BERTRAM, Rachael. The Surfing Coaching: Sources of Knowledge Acquisition. International Sport Coaching Journal, v. 5, n. 1, p. 1423, 2018.

COTÉ, Jean, et al. The coaching model: A grounded assessment of expert gymnastic coaches' knowledge. Journal of Sport and Exercise Psychology, v. 17, n. 1, p. 1-17, 1995.

CUSHION, Chris. Modelling the complexity of the coaching process. International Journal of Sports Science and Coaching, v. 2, n. 4, p. 395-401, 2007.

DUARTE, Tiago; CULVER, Diane M. Becoming a coach in developmental adaptive sailing: A lifelong learning perspective. Journal of Applied Sport Psychology, v. 26, n. 4, p. 441-456, 2014.

FUNOLLET, Feliu. Propuesta de clasificación de las actividades deportivas en el medio natural. Apunts: Educación Física y Deportes, v. 41, n. 124-129, 1995.

GALVÃO, Taís Freire; PANSANI, Thais de Souza Andrade; HARRAD, David. Principais itens para relatar Revisões sistemáticas e Meta-análises: A recomendação PRISMA. Epidemiologia e Serviços de Saúde, v. 24, p. 335-342, 2015.

GILBERT, Wade D; TRUDEL, Pierre. Analysis of coaching science research published from 1970-2001. Research Quarterly for Exercise and Sport, v. 75, n. 4, p. 388-399, 2004.

GRAY, Paul; COLLINS, Dave. The adventure sports coach: all show and no substance? Journal of Adventure Education and Outdoor Learning, v. 16, n. 2, p. 160-171, 2016.

GÜNTHER, Hartmut. Pesquisa qualitativa versus pesquisa quantitativa: esta é a questão? Psicologia: Teoria e Pesquisa, v. 22, n. 2, p. 201-210, 2006.

ICCE. International sport coaching framework. 2013. 55 p.

JONES, Robyn L; WALLACE, Mike. Another bad day at the training ground: Coping with ambiguity in the coaching context. Sport, Education and Society, v. 10, n. 1, p. 119-134, 2005.

JOWETT, Sophia. Coaching effectiveness: The coach-athlete relationship at its heart. Current Opinion in Psychology, v. 16, p. 154-158, 2017.

KIOSOGLOUS, Cameron; VIDIC, Zeljka. Shedding More Light on the Factors That Predict Coaching Success in Rowing. Journal of Sport Behavior, v. 40, n. 1, 2017. 
LANGAN, Edel; BLAKE, Catherine; LONSDALE, Chris. Systematic review of the effectiveness of interpersonal coach education interventions on athlete outcomes.

Psychology of Sport and Exercise, v. 14, n. 1, p. 37-49, 2013.

LORIMER, Ross; HOLLAND-SMITH, David. Why coach? A case study of the prominent influences on a top-level UK outdoor adventure coach. The Sport Psychologist, v. 26, n. 4, p. 571-583, 2012.

MACLELLAN, Justin; CALLARY, Bettina; YOUNG, Bradley W. Same coach, different approach? How masters and youth athletes perceive learning opportunities in training. International Journal of Sports Science and Coaching, v. 13, n. 2, p. 167-178, 2018.

MAHONEY, John W, et al. Implementing an autonomy-supportive intervention to develop mental toughness in adolescent rowers. Journal of Applied Sport Psychology, v. 28, n. 2, p. 199-215, 2016.

MILISTETD, Michel, et al. Coaching and coach education in Brazil. International Sport Coaching Journal, v. 1, n. 3, p. 165-172, 2014.

MILLAR, Sarah-Kate; OLDHAM, Anthony RH; DONOVAN, Mick. Coaches' self-awareness of timing, nature and intent of verbal instructions to athletes. International Journal of Sports Science and Coaching, v. 6, n. 4, p. 503-513, 2011.

MILLAR, Sarah-Kate, et al. Athlete and coach agreement: Identifying successful performance. International Journal of Sports Science and Coaching, v. 12, n. 6, p. 807-813, 2017.

MOTA, Cahê; SEDA, Vicente. Jogos de Tóquio 2020 terão escalada, surfe, skate, caratê e beisebol/softbol. 2016. Disponível em: http://globoesporte.globo.com/olimpiadas/ noticia/2016/08/jogos-de-toquio-2020-terao-escalada-surfe-skate-carate-e-beisebolsoftbol. html. Acesso em 09 maio 2019.

MOURA, Diego Luz; FERREIRA, Marcos Santos; SOARES, Antonio Jorge Gonçalves. Compreender, relativizar e desconstruir: os discursos do risco na produção acadêmica sobre esportes de aventura. LICERE, v. 19, n. 1, p. 1-20, 2016.

NELSON, Lee; GROOM, Ryan; POTRAC, Paul. Research methods in sports coaching. London: Routledge, 2014.

OLIVERA-BETRÁN, Alberto; OLIVERA-BETRÁN, Javier. Propuesta de una classificación taxonómica de las actividades físicas de aventura en la naturaleza: marco conceptual y análisis de los criterios elegidos. Educación Física y Deportes, v. 41, p. 108-123, 1995.

PAIXÃO, Jairo Antônio da, et al. Risco e aventura no esporte na percepção do instrutor. Psicologia \& Sociedade, v. 23, p. 415-425, 2011.

POPE, Clive C.; PENNEY, Dawn; SMITH, Tiaki B. Overtraining and the complexities of coaches' decision-making: managing elite athletes on the training cusp. Reflective Practice, v. 19 , n. 2, p. 145-166, 2018.

RAMOS, Valmor et al. A aprendizagem profissional-as representações de treinadores desportivos de jovens: quatro estudos de caso. Motriz, v. 17, n. 2, p. 280-291, 2011.

RAMOS, Valmor et al. Trajetória de vida de treinadores de surfe: análise dos significados de prática pessoal e profissional. Pensar a Prática, v. 17, n. 3, 2014.

RAMOS, Valmor; BRASIL, Vinicius Zeilmann; GODA, Ciro. A aprendizagem profissional na percepção de treinadores de jovens surfistas. Journal of Physical Education, v. 23, n. 3, p. 431-442, 2012. 
RAMOS, Valmor; BRASIL, Vinicius Zeilmann; GODA, Ciro. O conhecimento pedagógico para o ensino do surf. Journal of Physical Education, v. 24, n. 3, p. 381-392, 2013.

SAURY, Jacques; DURAND, Marc. Practical knowledge in expert coaches: On-site study of coaching in sailing. Research Quarterly for Exercise and Sport, v. 69, n. 3, p. 254-266, 1998.

SIMON, Scott; COLLINS, Loel; COLLINS, Dave. Observational Heuristics in a Group of High Level Paddle Sports Coaches. International Sport Coaching Journal, v. 4, n. 2, p. 235245, 2017.

THORPE, Holly; WHEATON, Belinda. 'Generation X Games', action sports and the Olympic movement: Understanding the cultural politics of incorporation. Sociology, v. 45, n. 5, p. 830-847, 2011.

TONG, Allison; SAINSBURY, Peter; CRAIG, Jonathan. Consolidated criteria for reporting qualitative research (COREQ): a 32-item checklist for interviews and focus groups. International Journal for Quality in Health Care, v. 19, n. 6, p. 349-357, 2007.

VIRGÍLIO, Ana Carolina Seragi, et al. Aprendizagem de treinadores esportivos: fontes de conhecimento e prática profissional nos jogos esportivos coletivos. Journal of Sport Pedagogy and Research, v. 3, n. 2, p. 20-26, 2017.

WALKER, Lauren F; THOMAS, Rebecca; DRISKA, Andrew P. Informal and nonformal learning for sport coaches: A systematic review. International Journal of Sports Science \& Coaching, v. 13, n. 5, p. 694-707, 2018.

WENGER, Etienne. A social theory of learning. Contemporary theories of learning, p. 209218, 2009.

WHEATON, Belinda. Introduction: Mapping the lifestyle sport-scape. Understanding Lifestyle Sport: Routledge, 2004. p. 13-40.

\section{Apoio:}

O presente trabalho foi realizado com apoio da Coordenação de Aperfeiçoamento de Pessoal de Nível Superior - Brasil (CAPES) - Código de Financiamento 001. This study was financed in part by the Coordenação de Aperfeiçoamento de Pessoal de Nível Superior Brasil (CAPES) - Finance Code 001. 